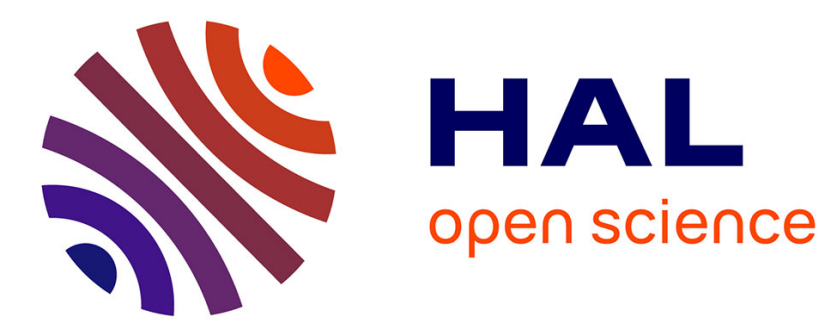

\title{
Optimization-Based Control Approaches to Humanoid Balancing
}

\author{
Aurélien Ibanez, Philippe Bidaud, Vincent Padois
}

\section{To cite this version:}

Aurélien Ibanez, Philippe Bidaud, Vincent Padois. Optimization-Based Control Approaches to Humanoid Balancing. Humanoid Robotics: A Reference, 2017, 10.1007/978-94-007-7194-9_71-1 . hal01711502

\section{HAL Id: hal-01711502 \\ https://hal.science/hal-01711502}

Submitted on 18 Feb 2018

HAL is a multi-disciplinary open access archive for the deposit and dissemination of scientific research documents, whether they are published or not. The documents may come from teaching and research institutions in France or abroad, or from public or private research centers.
L'archive ouverte pluridisciplinaire HAL, est destinée au dépôt et à la diffusion de documents scientifiques de niveau recherche, publiés ou non, émanant des établissements d'enseignement et de recherche français ou étrangers, des laboratoires publics ou privés. 


\title{
1 Optimization-based control approaches to humanoid balancing
}

\author{
Aurélien Ibanez ${ }^{1}$, Philippe Bidaud ${ }^{1,2}$, Vincent Padois ${ }^{1}$ \\ 1 - Sorbonne Universités, UPMC Univ Paris 06, CNRS UMR 7222, Institut des Systèmes \\ Intelligents et de Robotique (ISIR), 4 place Jussieu, 75252 PARIS cedex 05, France \\ 2 - ONERA F-91123 Palaiseau, France
}

\begin{abstract}
Summary. Balance is an essential feature of humanoids but, despite a strong understanding of its laws and dynamics, it remains an open problem for control applications. Optimizationbased control approaches explicitly include balance dynamics and constraints in the control problem in order to capture at best the behavior of the system and fully exploit it to reach complex control objectives. Although theoretically appealing, these approaches intrinsically induce a significant computational burden. In practice, this implies to resort to simplifications on the model and problem complexities, which limits the capacity to actually generate complex behaviors. In this chapter, an overview of the balance problem is first proposed. A general, abstract formulation of the balance control problem as an optimal control one is then derived. Three major approaches can be found in the literature, coping with the computational complexity of the general balance optimization problem. They range from offline motion planning to reactive whole-body control, and are presented in the remainder of the chapter.
\end{abstract}

Key words: humanoid balancing, motion planning, model predictive control, whole-body control

\subsection{Introduction}

Balance is a necessary condition in all use cases of humanoid robots as illustrated in figure 1.1 "Ensuring balance" is consequently a core feature of humanoid controllers and most of them rely on an explicit model of balance or on balance criteria extracted from these models.

However, balance is a complex notion and accounting for it in a general and efficient way at the control level is complex. Part of this complexity lies in the high dimensionality, nonlinearity, underactuation and hybrid nature of humanoid systems. These characteristics make it difficult to define computationally-friendly models of balance. This is all the more true that the impact of some given control action on balance cannot necessarily be anticipated for in a reactive way. Indeed, consequences of control actions have to be previewed over a time horizon to conclude on their compatibility with the general balance objective. As an example, unilateral contacts between the feet and ground provide the ability to modify the contacts configuration and generate feet motions with respect to the world, in other words locomotion. In these situations, breaking or making contact is a discrete action which consequences are a function of the next actions of the corresponding foot: if the center of mass of the overall system is launched forward but the flying foot never goes back to the ground the robot will 

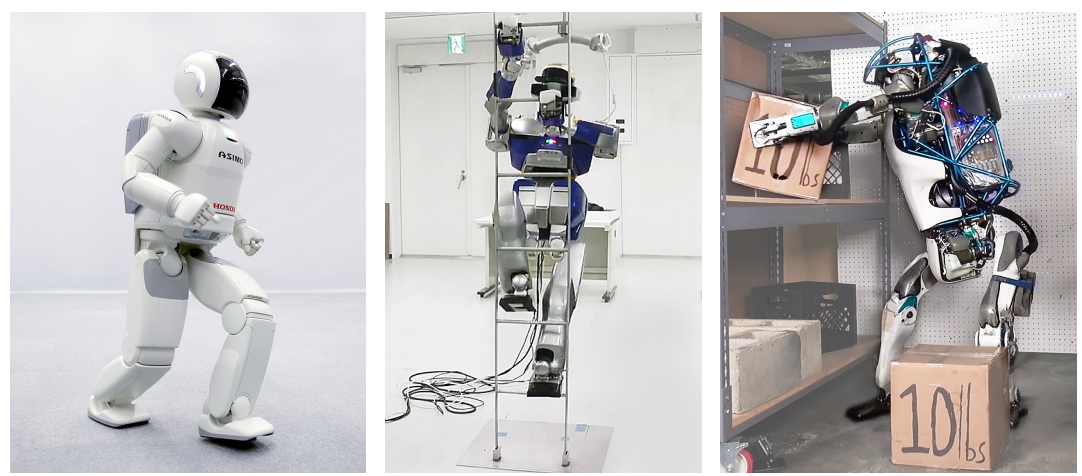

Fig. 1.1. Current state of the art of balancing humanoids. From left to right: Asimo, HRP-2 and Atlas robots. Courtesy of Honda Robotics, CNRS-AIST JRL, and Boston Dynamics.

inevitably fall whereas a proper next step may lead to dynamic equilibrium and balanced locomotion.

In scenarios where locomotion or corrective steps can be safely excluded, balance can be accounted for at the whole-body reactive control level (see figure 1.2), i.e. without the need for preview. In all other cases, predictions are required and, in the humanoid control literature, balance is most of the time first accounted for at higher abstraction and control levels (see figure 1.2). At the planning level, balance-compliant reference trajectories are computed in an optimal fashion over the complete time horizon. This is of course computationally costly and cannot be performed in real time, in closed-loop. At the task regulation level, the computational burden is solved by only considering some reduced part of the system's dynamics. This allows to refine the reference trajectories in a quasi real time way, accounting for the current state of the robot.

Balance models induce strong non-linearities such as the ones due to contact conditions. Moreover, unilateral contacts can be broken and discrete events (contact making/breaking) have to be accounted for at the control level. As a consequence, the control problems to be solved do not necessarily possess closed-form solutions and their solution space may not be convex and fully connected. These features advocate for a formulation of the control problems as constrained optimization ones. While at the planning and task regulation levels, the problems are often naturally posed as optimization ones, there has also been a shift toward optimization based methods at the whole-body reactive control level: historical contributions [28] and their successors [53, 9] in this domain did not account for inequality constraints in an optimal way and the last decade has seen a important growth of alternative, optimization-based, formulation of the control problem.

While several review works provide a very complete, locomotion-centered, view of the balance problem [23, 59], this chapter looks at the balance problem through the prism of optimization. It is organized as follows: First, the dynamics of free-floating systems are introduced and the non-linearities raised by contact mechanics are highlighted. Second, a general formulation of optimization-based approaches to the balance control problem is proposed to expose the computational challenges it induces. Levers classically employed to tackle these challenges are then identified. The prevalent balance indicators and metrics, 


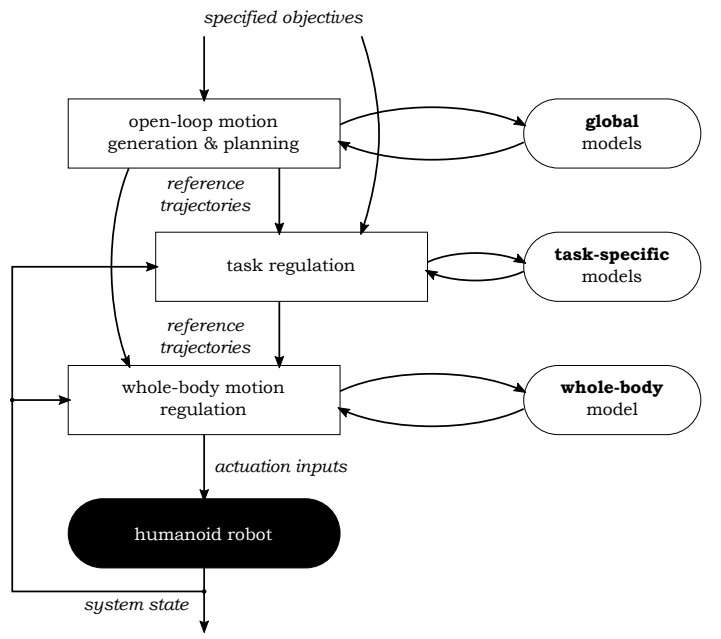

Fig. 1.2. Generic overview of control architectures

needed to describe control objectives and constraints, are therefore presented. An overview of optimization-based control approaches is finally proposed. These approaches are organized with respect to the level of abstraction at which they are employed to solve the balance control problem.

\subsection{Dynamics equations}

Efficient balance control requires the exploitation of the whole-body dynamics of the system. Within this perspective complexity rapidly arises from the high-dimensionality and nonlinearity of these dynamics. Indeed, motions of legged robots are fundamentally supported by the contact wrenches from their environment, wrenches generally being subject to the complex mechanical laws of unilateral contacts.

\subsubsection{Lagrangian equations of motion}

Legged robots are generally modeled from the control point of view as systems composed of rigid bodies, arranged in a tree structure with a base body as their root, called floating-base. The displacement of the robot in space is captured with respect to the position and orientation of a reference frame $\mathscr{R}_{b}$ attached to this body, with respect to a given reference inertial frame $\mathscr{R}_{0}$, called world frame. Being free-floating systems, the base is henceforth treated as linked with a 6-DoF virtual unactuated joint to the world, defining the pose $q_{b} \in S E(3)$ of $\mathscr{R}_{b}$ with respect to $\mathscr{R}_{0}$, with $S E(3)$ the special Euclidean group, as illustrated in figure 1.3 The associated twist $v_{b}$ is in $\mathbb{R}^{6}$.

The equations of motion for such systems can be derived [47] from the Lagrange formalism and take the form 


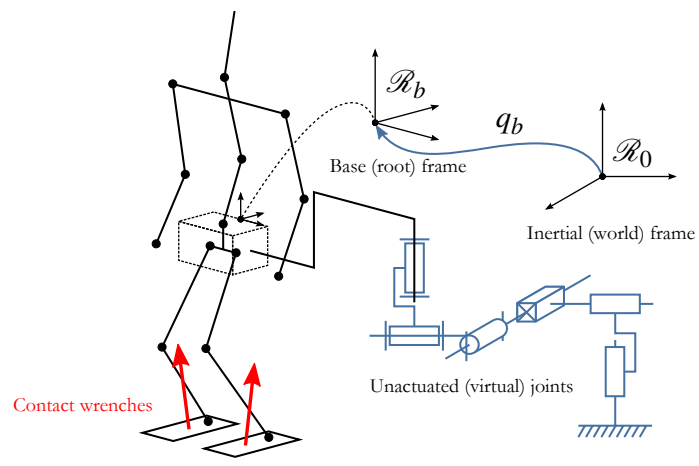

Fig. 1.3. Kinematic representation of floating-base systems. The root body of the tree structure of the mechanism is free-floating in a reference inertial frame $\mathscr{R}_{0}$.

$$
\underbrace{\left[\begin{array}{cc}
\mathbf{M}_{b} & \mathbf{M}_{b j} \\
\mathbf{M}_{b j}^{T} & \mathbf{M}_{j}
\end{array}\right]}_{\mathbf{M}(q)} \underbrace{\left[\begin{array}{c}
\dot{v}_{b} \\
\ddot{q}_{j}
\end{array}\right]}_{\dot{v}}+\underbrace{\left[\begin{array}{c}
n_{b} \\
n_{j}
\end{array}\right]}_{n(q, v)}+\underbrace{\left[\begin{array}{c}
g_{b} \\
g_{j}
\end{array}\right]}_{g(q)}=\left[\begin{array}{c}
\mathbf{0}_{6} \\
\mathbf{S} \tau
\end{array}\right]+\gamma_{c},
$$

where $q$, called generalized coordinates, parameterizes the configuration of the free-floating system. For the sake of simplicity, joints of the tree structure are assumed 1 to evolve in linear configuration spaces in this chapter: that is, $q_{j} \in \mathbb{R}^{n}$ parameterizes the joint configurations in the joint space $\mathbb{R}^{n}$, with $n$ the degree of freedom of the tree structure, and $q \in S E(3) \times \mathbb{R}^{n}$. The system velocity is subsequently represented with $v \in \mathbb{R}^{n+6}$, concatenating the floating-base twist $v_{b}$ and the generalized joint velocities $\dot{q}_{j} \in \mathbb{R}^{n}$. M is the kinetic energy - or mass - matrix of the system and $n$ and $g$ are respectively the vectors of non-linear effects (Coriolis and centrifugal) and of gravity, in the generalized coordinates space. Indices $\bullet_{b}, \bullet_{j}$ and $\bullet_{b j}$ denote definitions with respect to the base, the joints and both, respectively. $\mathbf{S} \in \mathbb{R}^{n \times n_{a}}$ is a matrix representing the actuation characteristics, with $n_{a} \leq n$ the number of actuated degrees of freedom, and $\tau \in \mathbb{R}^{n_{a}}$ is the actuation vector in the generalized coordinates relative to the joints. Vector $\gamma_{c}$ captures wrenches applied to the system from the environment in the generalized coordinates space.

Equations 1.1 clearly exhibits that, the floating-base being unactuated i.e. $\operatorname{dim}(\dot{v})>$ $\operatorname{dim}(\tau)$, legged robots are underactuated. It therefore appears that external wrenches $\gamma_{c}$ play a prevalent role in the dynamics of the floating-base, that is the 6 first lines of equation (1.1.

\subsubsection{Newton-Euler equations of motion}

The 6 first lines of equation (1.1) are differential equations related to the floating-base describing the dynamics of the system as a whole. Written at the center of mass of the system, they yield the Newton-Euler equations of motion

$$
\left[\begin{array}{c}
\dot{L}_{x} \\
M \ddot{x}
\end{array}\right]=w_{x}^{c}+w_{x}^{g}
$$

\footnotetext{
${ }^{1}$ Legged robots being generally articulated around revolute joints, this assumption holds.
} 
where $w^{c}$ and $w^{g}$ are respectively the net wrenches ${ }^{2}$ issued from contact and gravity effects, $M$ is the total mass of the system, and $L_{x}$ is the angular momentum of the system at its center of mass $x$. The Newton equation is the one related to linear momentum whereas the Euler one is related to angular momentum. These two equations plainly display the relation between contact wrenches and the dynamics of the center of mass, as from the structure of (1.1) actuation has no influence at this level. In the Newton equation, motion of the center of mass directly relates to the amplitude of external wrenches while the Euler equation captures their distribution.

It can be noted that differential equations 1.1 and $(1.2)$ are generally constrained. Indeed, inputs $\tau$, joint configurations $q_{j}$ and their temporal derivatives are generally bounded by technological limits. Laws of contact mechanics might additionally impose constraints on the contact wrenches $\gamma_{c}$ and, subsequently, on the system configuration and velocity and acceleration $(q, v, \dot{v})$.

\subsubsection{Contact mechanics}

External wrenches $\gamma_{c}$ in equation 1.1 are in the general case the result of contact forces between the system and its environment. A classical contact description is to consider nonadhesive contacts solely : contact forces are unilateral, i.e. they solely oppose penetration between the bodies in contact. With $\mathrm{n}$ the normal to the contact surface and $f_{c}$ a contact force on a body, the unilaterality condition writes

$$
f_{c}^{n} \geq 0, \quad \text { with } f_{c}^{n} \triangleq f_{c} \cdot \mathrm{n} .
$$

This characteristic 1.3 is not the only source of complexity : standard contact models indeed define contact laws as conditional equations, describing distinct possible contact cases. These contact cases involve both normal and tangential components of contact forces. In rigid body mechanics, which is the most widespread framework for multibody legged robots, normal contact mechanics are described as follow 3

$$
\begin{cases}f_{c}^{n} \geq 0 & \text { if contact } \\ f_{c}^{n}=0 & \text { otherwise }\end{cases}
$$

Tangential contact mechanics are more directly related to contact dynamics. Indeed, friction plays a role when a relative motion of the bodies in contact is involved. A standard friction model is the Coulomb dry friction model which states, with $\dot{x}$ the relative velocity of the bodies

$$
\left\{\begin{array} { r l } 
{ f _ { c } ^ { t } = \mu _ { c } f _ { c } ^ { n } \frac { \dot { x } ^ { t } } { \| \dot { x } ^ { t } \| } } & { \Leftarrow \| \dot { x } ^ { t } \| > 0 } \\
{ \| f _ { c } ^ { t } \| \leq \mu _ { c } f _ { c } ^ { n } } & { \Rightarrow \| \dot { x } ^ { t } \| = 0 }
\end{array} \quad \text { with } \left\{\begin{array}{l}
f_{c}^{t} \triangleq f_{c}-f_{c}^{n} \mathrm{n}, \\
\dot{x}^{t} \triangleq \dot{x}-(\dot{x} \cdot \mathrm{n}) \mathrm{n} .
\end{array}\right.\right.
$$

The dimensionless scalar $\mu_{c}$ is a parameter of the model, called coefficient of friction. The Coulomb model describes two regimes as illustrated in figure 1.4 in the kinetic friction, or sliding, regime the tangential contact force is given ; however, in the static friction, or sticking, regime this component is set-valued.

Rigid-body models also induce non-smooth dynamics: discontinuities in the relative velocity of body entering contact, impacts, can indeed occur.

\footnotetext{
${ }^{2}$ in $6 \mathrm{D}$ vector notation

${ }^{3}$ It can be noted that these contact mechanics are fully compatible with the unilateral contact condition 1.3 .
} 


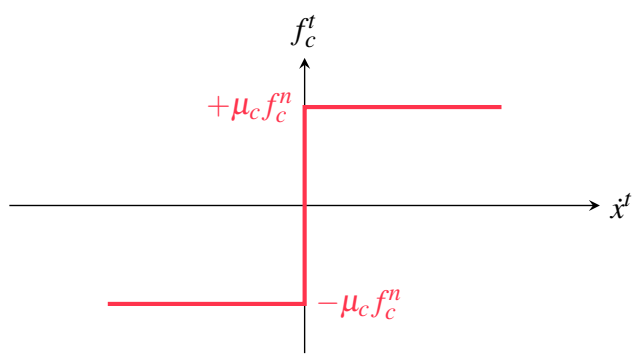

Fig. 1.4. Non-smooth and nonlinear contact dynamics described by Coulomb's friction law.

The dynamics of multibody legged robots present several sources of complexity to tackle at the control level. First, their dynamics state is described by variables of high dimensionality. Second, despite this high degree of freedom these systems are underactuated, posing inverse dynamics as an ill-posed problem and involving contact forces as a determining variable. Last, the dynamics governing these forces are non-smooth and subject to constraints.

\subsection{Optimal control approach to balance}

\subsubsection{Balance?}

At the whole-body motion regulation level, a humanoid robot is said to be balancing if, given its joint space and contact state (joint space and contact points positions and velocities), the external dynamic wrench induced by gravitational, inertial effects and other applied external wrenches applied to it can be compensated for by proper contact forces and joint torques. Achieving balance requires to respect the equation of motion while being able to maintain the contact state, i.e. not tipping over and not sliding. Given some tasks to perform, the global balance question boils down to some higher level problem where the evolution of the control input and of the state itself have to be determined to perform tasks at best while being able to reach a balanced terminal state. This general definition of balance includes jumping and running as potential types of balanced motions. However, in practice, ensuring balance at each time instant provides a better guarantee that a final balanced state can be achieved and jumping and running are generally not considered as a class of motion that can be achieved looking solely at the problem through the balance prism.

While this definition of balance can directly be translated into an optimization problem aiming at finding a solution (either at the global or at the regulation level), it does not say much about the existence of solutions. Indeed, as mentioned in the section "Stability analysis, not falling down" in [59], understanding the long term behavior requires to rely on stability and robustness notions. While many tools exist to define such notions for humanoids, the concept of viability is central. A viable state can be defined as one from which it can be guaranteed, over an infinite time horizon, that proper control will avoid the robot to lose balance. Ensuring the existence of a non empty and connected set of viable states and solving the control problem to maintain the future states of the system in this set is a very difficult, potentially intractable, problem. As a matter of fact, the balance literature offer much less 
ambitious visions of balance inducing limited behaviors but which guarantee balance in a tractable way.

This section describes potential ways of defining the optimization problem related to balance and recalls some of the balance indicators, criteria defined in the literature and which can be seen as special cases of a more general one.

\subsubsection{General control problem}

The control problem can be, in the most general way, expressed as the problem of finding a series of control inputs which will drive the system from an initial state towards objectives.

Reaching objectives The objective of the activity the system is involved into is denoted $\mathscr{F}^{d}$. Stating that the system has reached this objective can be written as

$$
\mathscr{F}(\mathscr{S})=\mathscr{F}^{d},
$$

where $\mathscr{S}$ denotes the state of the system, and $\mathscr{F}$ relates the state of the system to its outputs. In the general case however, there is no guarantee that this objective $\mathscr{F}^{d}$ is feasible nor that $\mathscr{F}$ is invertible. An intuitive and standard way to address this problem is to relax the constraint (1.6), and reformulate it as the least-squares problem with the introduction of the slack variable $\mathscr{W}$

which can be simplified to

$$
\begin{array}{ll} 
& \min _{\mathscr{S}, \mathscr{W}}\|\mathscr{W}\|^{2} \\
\text { s.t. } & \mathscr{F}(\mathscr{S})=\mathscr{F} d+\mathscr{W},
\end{array}
$$

$$
\min _{\mathscr{S}}\left\|\mathscr{F}(\mathscr{S})-\mathscr{F}^{d}\right\|^{2}
$$

where $\|\bullet\|$ is commonly the $\ell^{2}$-norm. Such an optimization-based formulation is therefore mainly driven by the need for finding solutions bringing the system as close as possible to its objectives, while explicitly taking into account the notion of infeasibility. Infeasibility can indeed be considered in the optimization problem with the constraints defining the set $\mathscr{K}^{\mathscr{S}}$ of admissible states over which the control problem is solved. That is, the objective-reaching problem is actually

$$
\min _{\mathscr{S} \in \mathscr{K}^{\mathscr{S}}}\left\|\mathscr{F}(\mathscr{S})-\mathscr{F}^{d}\right\|^{2}
$$

This constraint will nevertheless be ignored in the rest of this section for the sake of simplicity.

Problems 1.6 and 1.7 describe the problem of finding a state $\mathscr{S}$ of the system which achieves the desired objective $\mathscr{F}^{d}$. The control problem therefore consists in finding a trajectory of control inputs that will bring the system to this desired state.

Finding a way towards objectives In discrete time, let us describe the system as the dynamical system defined as

$$
\left\{\begin{array}{l}
\mathscr{S}_{k+1}=\mathscr{S}_{k+1}\left(\mathscr{S}_{k}, u_{k+1}\right) \\
\mathscr{F}_{k+1}=\mathscr{F}\left(\mathscr{S}_{k+1}\right)
\end{array}\right.
$$

In a dynamics framework, the system state at time $t_{i}$ hence depends on an anterior initial state $\mathscr{S}_{0}$ at time $t_{0}$ and on a history of control inputs $\mathscr{U}_{0, i} \triangleq\left[u_{1}, \ldots, u_{i}\right]^{T}$. This state at $t_{i}$ is denoted 


$$
\mathscr{S}_{i \mid 0} \triangleq \mathscr{S}_{i}\left(\mathscr{S}_{0}, \mathscr{U}_{0, i}\right)
$$

The control problem is therefore written, with an objective $\mathscr{F}^{d}$ to be reached at time $t_{f}$

$$
\min _{\mathscr{U}_{0, f}}\left\|\mathscr{F}\left(\mathscr{S}_{f \mid 0}\right)-\mathscr{F}^{d}\right\|^{2} .
$$

However, from the control point of view the dynamics of the environment and the system might be partially known. A feedback of the current system state $\mathscr{S}_{k}$ at each control time $t_{k}$ is therefore introduced in order to account for potential resulting disturbances, as illustrated in figure 1.5 . That is, the problem 1.9 is rewritten

$$
\forall t_{k}, \quad \min _{\mathscr{U}_{k, f}}\left\|\mathscr{F}\left(\mathscr{S}_{f \mid k}\right)-\mathscr{F}^{d}\right\|^{2},
$$

to be solved at each control time $t_{k}$, accounting for the current system state $\mathscr{S}_{k}$. Note that problem 1.10 can naturally be written in the optimal control canonical form, in continuous time.

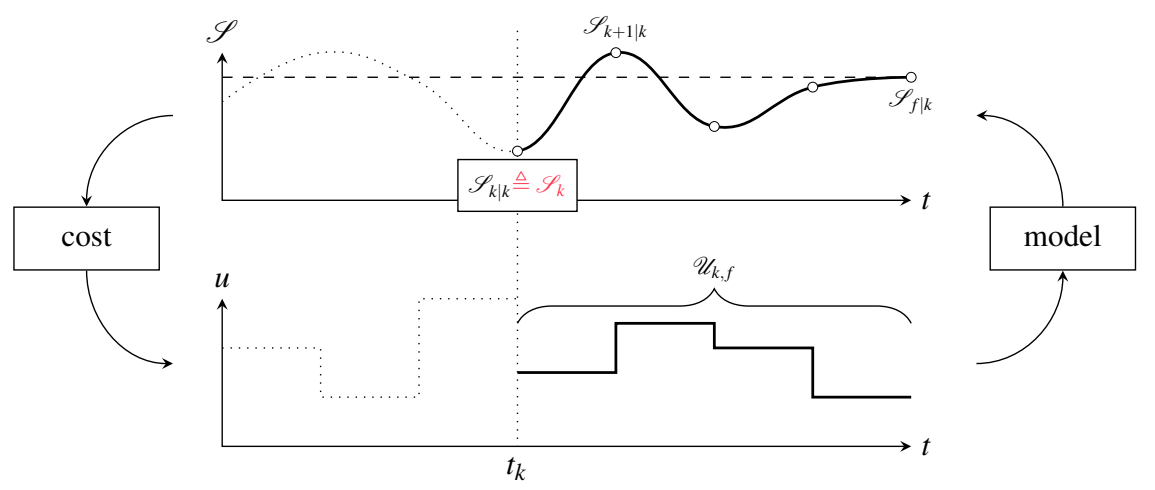

Fig. 1.5. Model-based optimal control problem over a time horizon with state feedback.

\subsubsection{The balance problem}

In the case of legged robots, final objectives $\mathscr{F}^{d}$ are generally considered as the outputs of balanced system states. Indeed, from a safety point of view balanced states are preferable as they are controllable [7], i.e. the system is able to handle disturbances as a path exists allowing to recover the desired state. With $\mathscr{K}_{k}^{\mathscr{B}}$ the set of balanced states at instant $t_{k}$, the problem 1.10 writes

$$
\begin{array}{ll}
\forall t_{k}, & \min _{\mathscr{U}_{k, f}}\left\|\mathscr{F}\left(\mathscr{S}_{f \mid k}\right)-\mathscr{F} d\right\|^{2}, \\
\text { s.t. } & \mathscr{S}_{f \mid k} \in \mathscr{K}_{f \mid k}^{\mathscr{B}} .
\end{array}
$$

The final constraint of problem 1.11) is nevertheless challenging as it involves the whole control history $\mathscr{U}_{k, f}$. A way to take on this challenge comes from the viability theory [60]: 
the control history $\mathscr{U}_{k, f}$ must induce system states which allow the system to maintain a balance state. While this problem is still intractable in the general case, a sufficient condition can yet be infered: if all intermediate states $\left\{\mathscr{S}_{k+1 \mid k}, \ldots, \mathscr{S}_{f-1 \mid k}\right\}$ are balanced, then a final state $\mathscr{S}_{f \mid k} \in \mathscr{K}_{f \mid k}^{\mathscr{B}}$ is reachable. Problem 1.11 is therefore rewritten

$$
\begin{array}{ll}
\forall t_{k}, \quad \min _{\mathscr{U}_{k, f}}\left\|\mathscr{F}\left(\mathscr{S}_{f \mid k}\right)-\mathscr{F} d\right\|^{2}, \\
\text { s.t. } & \mathscr{S}_{i \mid k} \in \mathscr{K}_{i \mid k}^{\mathscr{B}} \quad \forall i \in[k+1, f] .
\end{array}
$$

In the robot control framework, various sources of disturbances are present. For robustness, stability and feasibility considerations, the control problem 1.12 is hence usually reformulated in order to keep the system away from stability boundaries. With $\mathscr{B}$ a metrics capturing the distance of the system to the stability boundaries, this yields the new formulation

$$
\begin{aligned}
& \forall t_{k}, \quad\left\{\begin{array}{l}
\min _{\mathscr{U}_{k, f}}\left\|\mathscr{F}\left(\mathscr{S}_{f \mid k}\right)-\mathscr{F} d\right\|^{2}, \\
\max _{\mathscr{U}_{k, i-1}} \mathscr{B}\left(\mathscr{S}_{i \mid k}\right) \quad \forall i \in[k+1, f],
\end{array}\right. \\
& \text { s.t. } \quad \mathscr{S}_{i \mid k} \in \mathscr{K}_{i \mid k}^{\mathscr{B}} \quad \forall i \in[k+1, f] \text {. }
\end{aligned}
$$

\subsubsection{Challenges}

The control problem (1.13) raises several challenges to take on. First, the dimension of the control input history $\mathscr{U}_{k, f}$ can rapidly grow for legged robots and long-term objectives. Indeed legged robots present a high degree of freedom and the actuation vector $\tau$ is therefore of high dimension.

Second, the model presents strong non-linearities predominantly arising from the non-linear evolution of the dynamics model 1.1 with respect to the state $\mathscr{S}$ and from the non-smooth contact dynamics as discussed in paragraph 1.2 .3

Last, the optimization problem (1.13) is not convex in the general case. Indeed, balance constraints do not necessarily describe a convex set and no characteristic of the cost functions suggests the existence of a single, global optimum.

Additionally, the stabilization of the system dynamics and the regulation of disturbances require the control problem to be solved at a frequency consistent with the overall dynamics. In the case of legged robots, this frequency is commonly around $100 \mathrm{~Hz}$ to $1 \mathrm{kHz}$ which leads to the need for solutions every 1 to $10 \mathrm{~ms}$. This computational requirement, with respect to the current state of computer science and optimization techniques, prompts to consider different approaches to the control problem (1.13).

\subsubsection{Various approaches to the balance control problem}

From the preceding remarks, three main levers can be identified to reduce the computational complexity of the control problem (1.13): solving frequency, time horizon and model complexity. 
Solving frequency The most straightforward approach to this challenge is to directly relax the computational requirement by considering a lower rate of control.

An extreme case is offline planning, where problem (1.13) is solved once and for all from an initial state $\mathscr{S}_{0}$ at an initial instant $t_{0}$; that is

$$
\begin{array}{ll} 
& \left\{\begin{array}{l}
\min _{\mathscr{U}_{0, f}}\left\|\mathscr{F}\left(\mathscr{S}_{f \mid 0}\right)-\mathscr{F}^{d}\right\|^{2}, \\
\max _{\mathscr{U}_{0, i-1}} \mathscr{B}\left(\mathscr{S}_{i \mid 0}\right) \quad \forall i \in[1, f],
\end{array}\right. \\
\text { s.t. } \quad \mathscr{S}_{i \mid 0} \in \mathscr{K}_{i \mid 0}^{\mathscr{B}} \quad \forall i \in[1, f] .
\end{array}
$$

Since this approach implies an offline resolution of the problem, no computation requirements are to be met ; non-linear and non-convex optimization techniques can therefore be employed despite their relative computational inefficiency. Stochastic solvers are commonly setup in order to explore the whole solution space, but other non-linear solvers are also envisioned in cases where local optima are sufficient.

While this approach allows to find initial solutions to the control problem, the requirement for a fast, closed-loop controller is still present to stabilize the dynamics of the system and handle disturbances of small time scale. This approach is therefore employed at relatively high levels of control, such as open-loop motion generation or task regulation (c.f. figure 1.2).

Time horizon A practical approach to tract the control problem (1.13) in closed-loop is to consider a reduced time horizon ; one of the most widespread approaches consisting in the formulation of the control problem over one single control step. This approach requires the definition of instantaneous objectives $\mathscr{F}_{k}^{d}$ at each control time $t_{k}$ defined such that $\mathscr{F}_{f}^{d} \triangleq \mathscr{F}^{d}$.

$$
\begin{aligned}
\forall t_{k}, & \left\{\begin{array}{l}
\min _{u_{k}}\left\|\mathscr{F}\left(\mathscr{S}_{k+1}\right)-\mathscr{F}_{k+1}^{d}\right\|^{2}, \\
\max _{u_{k}} \mathscr{B}\left(\mathscr{S}_{k+1}\right),
\end{array}\right. \\
\text { s.t. } & \mathscr{S}_{k+1} \in \mathscr{K}_{k+1}^{\mathscr{B}} .
\end{aligned}
$$

The set of instantaneous objectives $\left\{\mathscr{F}_{0}^{d}, \ldots, \mathscr{F}_{f}^{d}\right\}$ is commonly the output of a planning process (c.f. figure 1.2 ).

At the instantaneous level, the dimensionality of the original control problem is evidently reduced. Furthermore, non-linearities in the dynamics model can be handled through linearization of the equation of motion 1.1 . Indeed, considering $\mathbf{M}(q) \approx \mathbf{M}, n(q, v) \approx n$ and $g(q) \approx g$ is a practical assumption generally accepted for high control rates. Similarly, non-linearities from the contact dynamics are commonly adressed by considering, at this instantaneous timescale, the contact regime as fixed and pre-defined by a higher control level.

This extreme reduction of the time horizon to a single time step is nevertheless not the only option to approach the control problem. Indeed, reducing the time horizon would require the definition of local balance constraints which are generally over-conservative with respect to the original problem (1.11) in order to ensure that the final state is balanced, thus producing potentially suboptimal solutions. To address this issue, recent approaches can be found in the literature considering an horizon of several time steps, in the Model Predictive Control framework commonly. Within this framework, the control problem writes 


$$
\begin{aligned}
& \forall t_{k}, \quad\left\{\begin{array}{l}
\min _{\mathscr{U}_{k, k+h}}\left\|\mathscr{F}\left(\mathscr{S}_{k+h \mid k}\right)-\mathscr{F}_{k+h}^{d}\right\|^{2}, \\
\max _{\mathscr{U}_{k, i-1}} \mathscr{B}\left(\mathscr{S}_{i \mid k}\right) \quad \forall i \in[k+1, k+h],
\end{array}\right. \\
& \text { s.t. } \quad \mathscr{S}_{i \mid k} \in \mathscr{K}_{i \mid k}^{\mathscr{B}} \quad \forall i \in[k+1, k+h] \text {, }
\end{aligned}
$$

where $h$ is a finite future time horizon.

However, the computational complexity of such approaches is still an open problem in legged robotics.

Model complexity The central barrier to solving practically the control problem 1.13 actually comes from the complexity of the model itself. This complexity mainly takes the form of dimensionality, non-linearities and non-smoothness. To overcome this obstacle, model reduction and simplification are commonly employed. At the lowest control level, model reductions or simplifications are usually avoided in order to compute actuation inputs with the utmost validity. However, higher control levels can largely benefit from a reduction of the model complexity, the complete dynamics model being enforced at the following control level (c.f. figure 1.2). A common approach in this perspective is to use this intermediate problem as an online trajectory generator.

Since the contact dynamics are one of the predominant sources of non-linearity and nonsmoothness, a relaxed, smooth approximation of these dynamics allows fast resolutions of the control problem [57] over a finite time horizon. Another common approach is to consider reduced dynamics, not subject to non-linearities or non-smoothness. For example, reducing the control problem to the center of mass and considering contact regimes as predefined allows to intuitively approximate linear models suitable for computationally-efficient control formulations [24 61].

\subsubsection{Balance indicators and criteria}

Within the control perspective described in the problem (1.13), appropriate definitions of the set of balanced states $\mathscr{K}^{\mathscr{B}}$ and of the balance metrics $\mathscr{B}$ are required.

One of the most commonly employed definitions relies on the Zero-Moment Point [58] (ZMP), or Center of Pressure (CoP). This point, defined without ambiguity for coplanar contact surfaces solely, allows to capture the net distribution of contact forces. If the CoP is strictly within the boundary of the support polygon, the contact surface cannot rotate around its edges, thus preventing tip-over situations. In other terms, if this condition is respected a torque can be produced at the contact level in order to produce a balancing motion. This condition is widely used as the definition of the set of balanced states $\mathscr{K}^{\mathscr{B}}$ in balance controllers. A definition of a balance metric $\mathscr{B}$ is also generally derived from this condition as the distance between the CoP and the boundaries of the support polygon. However, this condition is not necessary : it is indeed over-conservative as stable dynamic walking motions can be produced with the CoP reaching the edges of the support polygon, with point feet for example. Nevertheless, its efficiency has been largely proved for flat-foot dynamic balance and walking.

A related yet more general form of the ZMP is introduced by Goswami in [15] as the Foot Rotation Indicator (FRI). Defined as the point on the ground where the net ground reaction force would have to act to keep the foot stationary, it can cross the boundaries of the support polygon if the foot is experiencing rotational accelerations. Therefore, it provides a metric 
capturing both positive and negative distances to the stability margin.

The ZMP and FRI indicators however lack genericity as being specifically designed for biped locomotion scenarios. Although generalizations of these indicators can be found in the literature to extend them to more complex multi-contact situations, reverting to the essential definition of balance provides practical solutions to the definition problem of balanced states and metrics.

One the one hand, an instantaneous approach to balance considers the system to be dynamically balanced if there exist admissible contact forces that can support its motion, as illustrated in figure 1.6 Regarding the system as a whole, it essentially states that the NewtonEuler equations of motion (1.2) and contact mechanics are satisfied. A balance stability margin can henceforth be defined as the quantification of either the admissible motions around the current state or the disturbance wrenches that can be supported [2].

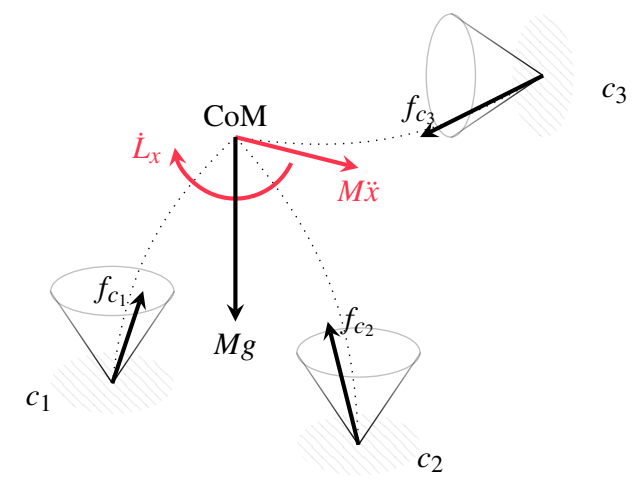

Fig. 1.6. The system is instantaneously balanced if there exist admissible contact forces $f_{c_{i}}$ that can support its motion $\left(\dot{L}_{x}, M \ddot{x}\right)$.

On the other hand, a long-term approach to balance requires the consideration of viability. Pratt and Tedrake propose to this aim to approach viability through the notion of capturability in [52]. A state is said to be capturable if it is controllable to stable fixed-points, i.e. a stable state with a null kinetic energy can be reached from this state. The definition of the Capture Point (CP) is derived from this approach as the point that the CoP should reach in order to bring the system to a capturable state ; the $\mathrm{CP}$ must therefore lie withing the support polygon in order to be reachable by the CoP. The Capture Point is not unique, and describes a capture region. A stability metric can therefore be derived as the overlap between the capture region and the support polygon, capturing the ability of the system to reach stabilizing states.

\subsection{Optimizing balance at the planning level}

Whole-body motion planning consists in generating open-loop joint space trajectories and, potentially, associated control inputs with the overall goal of reaching prescribed objectives 5

\footnotetext{
${ }^{4}$ These objectives can be described in the most general way in terms of operational space objectives for some body-part of the robot, e.g. its hands.
} 
This motion generation problem is subject to constraints related to the physics of the system both in terms of equation of motion and limits on various meaningful variables (joint position, velocity and torque, distance to obstacles, interaction wrenches, contact points,...). These constraints encapsulate the notion of balance.

Looking at this problem, it appears to be strongly non-linear and non convex both in terms of the overall cost function to optimize and the constraints to consider. The computational cost is thus important and highly sensitive to the degree of accuracy of the retained models. One way to address these types of optimization problem is to resort to stochastic approaches aiming at finding feasible and potentially locally optimal solutions through smart or random sampling. Another approach consists in applying non-linear optimization techniques leading to locally optimal solution as well but which are generally often influenced by an initial guess. As a matter of fact, the literature in this domain can clearly be divided into what can be called stochastic and non-stochastic whole-body motion planning even if many contributions mix both approaches. Some essential contributions in both domains are presented hereafter with a focus on the way the computational cost problem is addressed. Indeed, being able to plan fast is a desirable property.

\subsubsection{Stochastic whole-body motion planning}

Stochastic planning methods generally rely on sampling-based approaches used to explore the configuration space in order to find a feasible (constraint compliant) path from a starting configuration to a final one [33]. When dynamic constraints such as the ones imposed by balance come into play, a first possible approach can be to apply similar exploration techniques in the state space. However, doing so severely increases the dimensionality of the search problem which is already large with humanoids. Alternatively, a decoupled approach can be adopted where the problem is first solved at the kinematic level. The obtained path is then transformed in order to account for dynamic constraints. This is the approach retained in the pioneering work in [31] where a collision free path is first computed using a standard randomized planner and then optimized under constraints in order to enforce constraints upon the center of gravity projection and zero moment point trajectory in order to maintain balance. In this work, only feet contacts are considered and the contact state (stance) remains constant. The problem of finding a sequence of feasible supportive contacts and associated joint space configurations and contact forces is thus not tackled. To tackle this more complex problem, the methodology proposed in [16] also rely on a two-stage approach but the problem separation is of a different nature. Indeed, the first level of search is done at the stance level, trying to find a sequence of kinematically and statically feasible stances (with no restriction on the coplanarity of the feet contacts). This search is biased by a pre-computed motion primitives library aiming at improving the quality of the produced motions. These primitives are also used at the second level where a feasible path is found in configuration space between two consecutive stance transitions. The work in [5] builds on this approach to tackle multi-contact $(>2)$ problems. At first, similarly to [31], a collision free guide path is built using standard configuration space path planning techniques. While this path is collision free, it tries to minimize the distance to obstacles that could serve as potential supportive contacts. Then, a sequence of kinematically and statically feasible stances is searched for using an iterative, best-first algorithm. This algorithm favours stances inducing configurations close to the guide path. Kinematic and static feasibility is checked for using a non-linear constrained optimization solver outputting an optimal joint space configuration and optimal contact forces under static balance and collision avoidance constraints. The found solution aims at minimizing the distance to the pre-planned 
guide path, the required torque and contact forces as well as some cost favouring transition to the next stance, and involves large computation times $\left(10-20 \mathrm{~min}\right.$, Intel ${ }^{\circledR}$ Pentium ${ }^{\circledR}$ IV @ $3 G H z)$. At run time, an optimization based multi-objective whole body controller is used to ensure a dynamic execution of the overall motion while stance switching are monitored using a finite-state machine. The work in [7] follows a similar two-stage logic and proves that any statically balanced, collision-free path for a legged robot sliding on the ground can be approximated by a dynamically balanced, collision-free walk trajectory. Even though it induces a limitation on the type of considered ground (planar), this property is exploited to simplify the search for a dynamically feasible trajectory and allows for significantly reduced computation times ( 2 min Intel $^{\circledR}$ Core2Duo $\left.{ }^{\circledR} @ 2 G H z\right)$.

\subsubsection{Non-stochastic whole-body motion planning}

Similarly to previous stochastic approaches, non-stochastic approaches do not directly tackle the whole-body planning problem without prior simplifications. In the seminal work presented in [45], periodic and symmetric running motions are studied on an 11 degrees of freedom planar humanoid model which however a full description of the hybrid dynamics problem related to contact switching. Contrarily to the general definition of balance used throughout this chapter, balance appears in this work as an objective, together with energy consumption and a cost on the terminal state of the system. This balance objective aims at reaching a stable limit cycle by minimizing the spectral radius of the Jacobian of the Poincaré map. The optimization variable is composed of the evolution of the state, control input and stance switching instants. Robot morphology parameters are also optimized, this work being developed with robot design concern in mind. The problem is solved using what can be seen as a tailored Sequential Quadratic Program (SQP) based on MUSCOD, a multiple shooting algorithm for direct solution of optimal control problem [3]. In [11] and [36] similar optimization problems are solved but an initial guess is provided as a collision free path obtained using randomized planning techniques and a sequence of feasible contact stances obtained using the work in [5] respectively. These initial guesses remove the need for considering a full description of the hybrid dynamics and more complex humanoids can be considered, especially as much less dynamic motions are considered, while still involving significant computation times (from several minutes, Intel ${ }^{\circledR}$ Core2Duo ${ }^{\circledR} @ 1.6 \mathrm{GHz}$, to several hours, Intel ${ }^{\circledR}$ Xeon ${ }^{\circledR} @ 2.5 \mathrm{GHz}$, respectively). More recently, a similar non-linear optimization approach is retained in [32] where the simplification at the planning level lie in the use of a full model description for kinematics constraints whereas only the free-floating dynamics is considered for the equation of motion. While the evolution of the state, contact locations, contact forces, stances transitions and center of mass dynamics can be computed, joint level torque inputs cannot be generated using such a technique. However the robot is assumed to have large actuation capabilities and the actual computation of the control inputs is performed at the reactive level using an optimization based multi-objective whole body controller.

\subsection{Optimal regulation of balance tasks}

Translating specified objectives into balance-consistent references is a first step towards the conservation of balance during the execution of activities. Nevertheless, unexpected disturbances from modeling errors or external perturbations must be considered in order to maintain 
balance. State feedback approaches are consequently employed to compute the actuation input tracking these references, while ensuring balance with a view to the current state of the system. The complexity of legged robots dynamics (1.1) however tends to lead to computationally inefficient formulations of the balance problem. A common approach is therefore to introduce an intermediate level refining open-loop references into closed-loop objectives.

To this aim, task regulators are introduced accounting for state feedback, while using reduced models to reduce the computational cost. In the case of balance, the most widespread reduced model capturing the dynamics of the system as a whole is described by the NewtonEuler equations of motion (1.2). Optimization-based approaches are commonly employed at this level of abstraction to additionally enforce balance constraints in a closed-loop formulation. Key formulations are presented in this section in order to apprehend common techniques to approach the balance problem at this level.

\subsubsection{Model Predictive Control}

A common optimal approach to balance regulation is Model Predictive Control. MPC employs a model of the system dynamics to preview its behavior over time and compute an optimal horizon of control inputs maintaining the system into the valid state space.

When writing the balance control problem over a future horizon as described in problem 1.13, several obstacles are met as it requires a time-integration of the local system model (e.g. the equations of motion (1.1)). Indeed, the relation between the local model parameters (e.g. the inertia matrix $\mathbf{M}$ ) and the local actuation input $u$ is generally nonlinear, leading to nonlinear and non-convex optimization problems. Integrating the local model furthermore implies the resolution of the contact forces which brings additional nonlinearities and the dimension of the actuation input further increases the computational cost of the problem. A straightforward approach to bypass these obstacles is to consider a reduced local model of the system in order to decrease its complexity and dimensionality. Additionally, considering the contact regimes as predefined over the problem horizon allows to discard the complexity raised by contact dynamics.

Employing a reduced model, such approaches therefore output optimal control inputs defined at a level of abstraction higher than the actual actuation of the system. These control inputs are thereafter used as refined reference trajectories to be tracked at the whole-body level.

ZMP-based formulations The interest for MPC techniques in the humanoid robotics literature was mainly initiated by Kajita et al. in the form of Preview Control [24]. In this work, the control problem is reduced to the center of mass of the system and is written over a finite time horizon as schematized in equation 1.16 . Considering the center of mass jerk as the control input at this level, a linear model can be induced under some assumptions. The main hypotheses of this formulation are that the center of mass keeps a constant altitude over the preview horizon and that the rate of change of angular momentum of the system is negligible. Balance is approached in this work through the ZMP model and criterion, with its common assumptions: contacts with the environment are coplanar, without any constraints on contact forces. The control problem in this formulation is solely written as the maximization of balance, defined as a tip-over risk with the ZMP criterion, through the computation of an optimal horizon of center of mass jerks. While this approach proves efficient in stabilizing the dynamics of the center of mass in walking motions, one of its major flaws is the lack of balance guarantee. 
Wieber therefore proposed [61] further developments of this formulation to account for constraints on the ZMP position and subsequently allow the consideration of stability margins.

The original, unconstrained Preview Control was in parallel subject to extensions to account for external disturbances from the environment. Kanzaki et al. for example propose in [26] to directly account for an expected external impacts on the robot in the reduced dynamics model. In [20] this approach is further developed to simultaneously optimize balance and manipulation tasks in order to maximize their respective performance with respect to known external forces applied to the hand of the robot. Another approach discards the constant CoM altitude assumption which allows to additionally include friction considerations in the balance problem [21]; the extra computational cost being reduced with the formulation of a distributed optimization problem.

These approaches nevertheless require the predefinition of a reference trajectory of the ZMP, defined through a prior choice of feet positions over the preview horizon ; feet positions being the outputs of a planning process for example. Also, in such formulations the optimization problem solely considers a balance objective, and no task objective is specified. To enhance the flexibility of the balance controller, developments were performed in order to include feet positions as variables of the optimization problem [10]. These additional degrees of freedom in the control problem allow to consider a task objective in the form of the tracking of a reference center of mass velocity, in addition to the maximization of balance. Besides, they can be exploited to recover from larger disturbances.

This approach has been subject to multiple developments. Stephens and Atkeson for example proposed in [56] to extend this formulation to a force-control framework. Herdt et al. preceded the control problem with an additional optimization problem aiming at computing optimal orientations of the feet in order to track an angular velocity with the trunk [18], thus improving the capacities of the controller to recover from large disturbances. The consideration of pre-defined stepping instants and durations allows to maintain a computationallyefficient formulation of the problem (QP) and thus involve real-time compatible computation times ( $<1 \mathrm{~ms}$ for an horizon of $1.6 \mathrm{~s}$ in 16 segments of $100 \mathrm{~ms})$. To further increase the flexibility of the balance controller and reduce the influence of a planning process, developments were also proposed in the recent literature to additionally consider the duration and instants of the steps in the optimization problem [22], at the expense of computational efficiency ( $<60 \mathrm{~ms}$ for an horizon of $1.5 \mathrm{~s}$ in 8 ! segments). Nonlinearities in the model are handled with the use of integer variables to capture the discrete nature of the model.

Other criteria MPC formulations based on the ZMP criterion have also been extended to other criteria, such as the capture point in [30]. A more generic approach can also be found in [17] where the balance problem is directly written as the optimization problem of contact forces acting on the system, thus allowing the consideration of multi-contacts scenarios. However, this genericity is obtained at the cost of computational impracticability. Similarly, Perrin et al. propose in [49] to consider multi-contacts scenarios.

\subsubsection{Instantaneous formulations}

Optimization-based approaches are nevertheless not restricted to MPC methods at this level. Open-loop references can also be refined at the instantaneous level and translated into instantaneous acceleration or force objectives. The computational burden being reduced with such formulations over a single step horizon, complex scenarios can be practically considered. 
With the objective of distributing a reference value of the balancing net contact wrench over multiple contact points, while respecting friction constraints, Ott et al. propose in [48] an optimization-based formulation of the balance problem focused on the center of mass dynamics. With this model, any contact configuration can be considered. The resulting problem is written as a $\mathrm{QP}$, aiming predominantly at minimizing the tracking error of the desired net wrench. This intermediate control problem outputs a set of contact forces, considered as objectives to be tracked at a lower-level for each contact points. Lee and Goswami similarly propose to focus on a model of the dynamics of the system reduced to its center of mass in [35] while considering each individual contact wrenches instead of a net contact wrench. This approach refines at this level desired rates of change in linear and angular momenta into admissible ones, with respect to the foot geometry and contact constraints. In order to keep the computational cost of this problem as low as possible, the authors perform a sequential distribution of the original optimization problem to obtain two successive QP problems.

\subsection{Optimization-based whole-body control}

Desired references and objectives, generally expressed at a relatively high level of abstraction, needs to be translated at the actuation level to compute the corresponding actuation control inputs.

\subsubsection{Local optimality}

A widespread optimal approach to balance at the actuation level is to solve the inverse kinematics or dynamics problem under balance constraints. At this level, balance constraints are generally restricted to the consideration of contacts stability and coordinated center of mass accelerations.

On the one hand, this problem has historically been solved using analytical methods based on null-space projection techniques and accounting for constraints as avoidance tasks. Nonetheless, this approach is doomed to fail: these constraints-related tasks aim at getting away from the constraints (active avoidance). The number of constraints being potentially higher than the number of DOF, they cannot lead to control solutions that can strictly guarantee the respect of all constraints. On the other hand, whole-body controller are written as a quadratic multi-objective optimization problem under linear constraints where priorities between the objectives can be dealt with through strict of soft hierarchy. The resulting optimization problem is therefore to compute optimal actuation inputs which maximize the tracking performance of desired references while respecting balance constraints. The logic behind this choice is straightforward. First, the equation of motion and joint space to task spaces mappings can be written as equalities but they are not sufficient to describe the overall dynamics and physical behavior of a robot. Indeed, other intrinsic physical constraint have to be accounted for at the joint level as well as in Cartesian space. These constraints do not solely describe relationships between physical quantities but also limits which cannot (control input saturation) or should never be crossed in order to maintain the robot and its environment in proper working conditions. Theses limits translate into inequalities. Assuming a convex solution space, the optimal solution of the control problem lie at the boundary of the feasible (constraint compliant) solution space. Finding the optimal solution thus boils down to finding the active constraint set, i.e. on which boundary it lies. Optimization problem solvers are designed to optimally choose this subset of constraints that should be considered when 
computing the optimal solution of the control problem. The strong mathematical background in convex optimization is such that optimization based methods mostly outperform analytical methods attempting to heuristically activate constraints.

In the seminal work in [13], an obstacle avoidance technique includes a control law structure based on a Quadratic Program (QP). Since then, control approaches relying on optimization tools such as Linear Quadratic Problem solver have emerged for virtual humans [1, 6]. For real humanoids, to deal with prioritized inequality constraints more easily, hierarchical quadratic programming (HQP) approaches use numerical QP solvers to solve a Hierarchical Quadratic Program[25]. The idea of HQP is to first solve a QP to obtain a solution for a higher priority task objective; and then to solve another QP for a lower priority task, without increasing the obtained minimum of the previous task objective. This prioritization process corresponds to solving at best lower-priority tasks in the null-space of higher-priority tasks. The HQP algorithm is applied for solving prioritized inverse dynamics and whole-body motion control under unilateral constraints [54]. It requires to solve as many QPs as priority levels, which can be quite time consuming. The computation cost of hierarchical inverse kinematics with inequality constraints is improved by an algorithm developed in [12], which permits real time control of the HRP-4 humanoid robot. Similar work is performed in [19] where a reduction of the equation of motion allows for real time control of the SARCOS humanoid robot.

Generally, for an approach based on strict hierarchy, the relative importance of one task with respect to another one of different priority level is parametrized in a binary way: either strictly higher or strictly lower (lexicographic order). However, in many contexts, organizing tasks by assigning them a lexicographic order is not generic, i.e. can have some limitations. First, a strict priority is just an extreme case of the relations of task importance levels. In fact, a task may not always have a strict priority over another one and it is usually difficult to define a strict hierarchy among some of the tasks. Second, strict priorities can sometimes be too conservative so that they may completely block lower-priority tasks. Third, a change in the task set, such as a swap of task priorities, may lead to discontinuity. An approach to smooth priority rearrangement between two tasks is proposed in [27, 50] and approaches for continuous and simultaneous transitions of multiple tasks are developed in [44, 34]. A specific inverse operator is proposed in [44] to ensure continuous inverse in the analytical computation of control laws. The approach presented in [34] is based on intermediate desired values in the task space. When applied to humanoids, the number of tasks and the state dimension are such that the computational cost implied by these approaches is too high for practical use.

Smooth task transitions can be easily achieved within a framework using a weighting strategy [1, 6, 4] by the continuous variation of task weights [55]. These control frameworks solve all the constraints and task objectives in one QP and provide a trade-off among task objectives with different importance levels. As the performances of higher priority tasks cannot be guaranteed by simply adjusting the weights of task objectives, a prioritized control framework is proposed in [39] to ensure the performance of a higher-priority task within a user defined tolerance margin. However, this approach handles priorities of only two levels. More recently the work [40, 37] proposes a Generalized Hierarchical Framework which allows to describe both soft and strict priority problems with smooth priority transitions.

Recently, the work in [41 42] has questioned the notion of priorities: while some priorities are needed for robustness and safety reasons, trying to achieve incompatible tasks at the reactive level does not make sense. Thus, on-line task optimization is needed in order to feed reactive whole-body controllers with references which are compatible one with another and more importantly with constraints, among which balance is a very important one. 


\subsubsection{Whole-body MPC}

While the combination of instantaneous whole-body approaches with reduced MPC task controllers provides the control system with the consideration of both a future horizon and the whole-body dynamics of the system, their sequential layout is necessarily suboptimal. MPC approaches are therefore gradually extended to whole-body control with the consideration of models capturing the system state at the joint level. Computational limitations nevertheless requires the setup of simplifications of the control problem. Indeed, as discussed in Sec. 1.5 . time-integration is one of the major challenges of MPC formulations.

A significant improvement in this direction was performed by Tassa et al. in [57]. The authors address computational issues in two ways in this work.

First, the resolution of the MPC optimization problem is considered over the whole duration of the activity. That is, the control problem solely aims at continuously driving the system towards an all-time minimum, rather than finding an optimum at each control step. To this aim a suboptimal solution solely is demanded at each control step, generally obtained through a single iteration of the optimization algorithm. It therefore allows the consideration of much more complex optimization problems : explicit formulations of models are indeed not required as an update for a given control input is solely needed. In this particular work, an iterative Linear Quadratic Gaussian optimizer, a variant of Differential Dynamic Programming, is setup to work over the joint actuation space. Computational issues nevertheless arise from the needs to update the model rapidly for an iteration of the optimizer.

To meet this challenge, the second contribution of this work is thus to propose a fast dynamics integrator. Following the remark that frictional contacts are mainly responsible for the dynamics computational burden, the authors consider continuous contact models ignoring the discontinuous contact dynamics and propose a cheap smooth model producing realistic behaviors for the time scales of the MPC problem.

Balance is simply approached in [57] through a static metric on the CoM. Despite the use of this highly conservative balance criterion, dynamic and complex behaviors involving the whole-body of the system are discovered to track a target pose of the torso. However this controller required a time slowdown of $\times 7$ with optimization iterations of $140 \mathrm{~ms}$, thus forbidding a direct real-time implementation. Koenemann et al. nevertheless described in [29] a real-time implementation of this controller using a double rate control architecture. In this work, the MPC problem is employed as a trajectory generator running at $\times 10$ the rate of the lower, tracking control loop.

Mordatch et al. in [46] also consider regularization of contact dynamics as a solution to the computational issues it raises. This approach is however fundamentally different ; indeed, in order to avoid the costly computation of contact states from control inputs (performed by the dynamics simulator in the works of Tassa et al.), they are explicitly considered in the optimization problem as control variables. More specifically, the activation and deactivation of contacts is written as thresholds over a continuous variable describing whether a contact should be activated or not. However, the resulting formulation is not computationally-efficient enough to allow real-time implementations.

\subsection{Open-problems}

While whole-body Model Predictive Control stands out as a promising solution to the general balance control problem, it also suffers from the need for compromises. Its potential is, in 
the current state of the art, hindered by the difficulty to efficiently integrate the non-linear dynamics of humanoid systems. Future developments in this direction will undoubtedly provide keys to widen the scope of humanoids applications.

\section{References}

1. Yeuhi Abe, Marco da Silva, and Jovan Popović. Multiobjective control with frictional contacts. In Proceedings of the 2007 ACM SIGGRAPH/Eurographics symposium on Computer animation, pages 249-258. Eurographics Association, 2007.

2. Sébastien Barthélemy and Philippe Bidaud. Stability measure of postural dynamic equilibrium based on residual radius. In Advances in Robot Kinematics: Analysis and Design, pages 399-407. Springer, 2008.

3. H. G. Bock and K.-J. Plitt. A multiple shooting algorithm for direct solution of optimal control problems. In Proceedings of the 9th IFAC World Congressn, pages 242-247, 1984.

4. Karim Bouyarmane and Abderrahmane Kheddar. Using a multi-objective controller to synthesize simulated humanoid robot motion with changing contact configurations. In Intelligent Robots and Systems (IROS), 2011 IEEE/RSJ International Conference on, pages 4414-4419. IEEE, 2011.

5. Karim Bouyarmane and Abderrahmane Kheddar. Humanoid robot locomotion and manipulation step planning. Advanced Robotics, 26(10):1099-1126, 2012.

6. Cyrille Collette, Alain Micaelli, Claude Andriot, and Pierre Lemerle. Dynamic balance control of humanoids for multiple grasps and non coplanar frictional contacts. In $\mathrm{Hu}$ manoid Robots, 2007 7th IEEE-RAS International Conference on, pages 81-88. IEEE, 2007.

7. Sébastien Dalibard, Antonio El Khoury, Florent Lamiraux, Alireza Nakhaei, Michel Taïx, and Jean-Paul Laumond. Dynamic walking and whole-body motion planning for humanoid robots: an integrated approach. The International Journal of Robotics Research, page 0278364913481250, 2013.

8. Robin Deits and Russ Tedrake. Footstep planning on uneven terrain with mixed-integer convex optimization. In Humanoid Robots (Humanoids), 2014 14th IEEE-RAS International Conference on, pages 279-286. IEEE, 2014.

9. Andrea Del Prete, Francesco Nori, Giorgio Metta, and Lorenzo Natale. Prioritized motion-force control of constrained fully-actuated robots:task space inverse dynamics. Robotics and Autonomous Systems, 63:150-157, 2015.

10. Holger Diedam, Dimitar Dimitrov, Pierre-Brice Wieber, Katja Mombaur, and Moritz Diehl. Online walking gait generation with adaptive foot positioning through linear model predictive control. In Intelligent Robots and Systems, 2008. IROS 2008. IEEE/RSJ International Conference on, pages 1121-1126. IEEE, 2008.

11. Antonio El Khoury, Florent Lamiraux, and Michel Taix. Optimal motion planning for humanoid robots. In Robotics and Automation (ICRA), 2013 IEEE International Conference on, pages 3136-3141. IEEE, 2013.

12. Adrien Escande, Nicolas Mansard, and Pierre-Brice Wieber. Hierarchical quadratic programming: Fast online humanoid-robot motion generation. The International Journal of Robotics Research, page 0278364914521306, 2014.

13. B. Faverjon and P. Tournassoud. A local based approach for path planning of manipulators with a high number of degrees of freedom. In Proceedings of the IEEE International Conference on Robotics and Automation, volume 4, pages 1152-1159, 1987. 
14. Perle Geoffroy, Nicolas Mansard, Maxime Raison, Sofiane Achiche, and Emo Todorov. From inverse kinematics to optimal control. In Advances in Robot Kinematics, pages 409-418. Springer, 2014.

15. Ambarish Goswami. Postural stability of biped robots and the foot-rotation indicator (fri) point. The International Journal of Robotics Research, 18(6):523-533, 1999.

16. Kris Hauser, Timothy Bretl, Jean-Claude Latombe, Kensuke Harada, and Brian Wilcox. Motion planning for legged robots on varied terrain. The International Journal of Robotics Research, 27(11-12):1325-1349, 2008.

17. Bernd Henze, Christian Ott, Maximo Roa, et al. Posture and balance control for humanoid robots in multi-contact scenarios based on model predictive control. In Intelligent Robots and Systems (IROS 2014), 2014 IEEE/RSJ International Conference on, pages 3253-3258. IEEE, 2014.

18. Andrei Herdt, Nicolas Perrin, and Pierre-Brice Wieber. Walking without thinking about it. In Intelligent Robots and Systems (IROS), 2010 IEEE/RSJ International Conference on, pages 190-195. IEEE, 2010.

19. Alexander Herzog, Ludovic Righetti, Felix Grimminger, Peter Pastor, and Stefan Schaal. Balancing experiments on a torque-controlled humanoid with hierarchical inverse dynamics. In Intelligent Robots and Systems (IROS 2014), 2014 IEEE/RSJ International Conference on, pages 981-988. IEEE, 2014.

20. Aurelien Ibanez, Philippe Bidaud, and Vincent Padois. Unified preview control for humanoid postural stability and upper-limb interaction adaptation. In Intelligent Robots and Systems (IROS), 2012 IEEE/RSJ International Conference on, pages 1801-1808. IEEE, 2012.

21. Aurelien Ibanez, Philippe Bidaud, and Vincent Padois. A distributed model predictive control approach for robust postural stability of a humanoid robot. In Robotics and $\mathrm{Au}$ tomation (ICRA), 2014 IEEE International Conference on, pages 202-209. IEEE, 2014.

22. Aurelien Ibanez, Philippe Bidaud, and Vincent Padois. Emergence of humanoid walking behaviors from mixed-integer model predictive control. In Intelligent Robots and Systems (IROS 2014), 2014 IEEE/RSJ International Conference on, pages 4014-4021. IEEE, 2014.

23. Shuuji Kajita and Bernard Espiau. Legged robots. In Springer handbook of robotics, pages 361-389. Springer, 2008.

24. Shuuji Kajita, Fumio Kanehiro, Kenji Kaneko, Kiyoshi Fujiwara, Kensuke Harada, Kazuhito Yokoi, and Hirohisa Hirukawa. Biped walking pattern generation by using preview control of zero-moment point. In Robotics and Automation, 2003. Proceedings. ICRA'03. IEEE International Conference on, volume 2, pages 1620-1626. IEEE, 2003.

25. Oussama Kanoun, Florent Lamiraux, Pierre-Brice Wieber, Fumio Kanehiro, Eiichi Yoshida, and Jean-Paul Laumond. Prioritizing linear equality and inequality systems: application to local motion planning for redundant robots. In Robotics and Automation, 2009. ICRA'09. IEEE International Conference on, pages 2939-2944. IEEE, 2009.

26. Shigeru Kanzaki, Kei Okada, and Masayuki Inaba. Bracing behavior in humanoid through preview control of impact disturbance. In Humanoid Robots, 2005 5th IEEERAS International Conference on, pages 301-305. IEEE, 2005.

27. F. Keith, N. Wieber, P.-B.and Mansard, and A. Kheddar. Analysis of the discontinuities in prioritized tasks-space control under discreet task scheduling operations. In Proceedings of the IEEE/RSJ International Conference on Intelligent Robots and Systems, pages 3887-3892, 2011.

28. Oussama Khatib, Luis Sentis, and Jae-Heung Park. A unified framework for whole-body humanoid robot control with multiple constraints and contacts. In European Robotics Symposium 2008, pages 303-312. Springer, 2008. 
29. J Koenemann, Andrea Del Prete, Yuval Tassa, E Todorov, Olivier Stasse, M Bennewitz, and Nicolas Mansard. Whole-body Model-Predictive Control applied to the HRP-2 Humanoid. In IEEE/RSJ International Conference on Intelligent Robots and Systems (IROS 2015), page 8p., Hamburg, Germany, September 2015.

30. Manuel Krause, Johannes Englsberger, Pierre-Brice Wieber, and Christian Ott. Stabilization of the capture point dynamics for bipedal walking based on model predictive control. In Robot Control, volume 10, pages 165-171, 2012.

31. James J Kuffner Jr, Satoshi Kagami, Koichi Nishiwaki, Masayuki Inaba, and Hirochika Inoue. Dynamically-stable motion planning for humanoid robots. Autonomous Robots, 12(1):105-118, 2002.

32. Scott Kuindersma, Robin Deits, Maurice Fallon, Andrés Valenzuela, Hongkai Dai, Frank Permenter, Twan Koolen, Pat Marion, and Russ Tedrake. Optimization-based locomotion planning, estimation, and control design for the atlas humanoid robot. Autonomous Robots, 40(3):429-455, 2015.

33. S. LaValle. Planning algorithms. Cambridge university press, 2006.

34. J. Lee, N. Mansard, and J. Park. Intermediate desired value approach for task transition of robots in kinematic control. IEEE Transactions on Robotics, 28(6):1260-1277, 2012.

35. Sung-Hee Lee and Ambarish Goswami. A momentum-based balance controller for humanoid robots on non-level and non-stationary ground. Autonomous Robots, 33(4):399_ 414, 2012.

36. Sébastien Lengagne, Joris Vaillant, Eiichi Yoshida, and Abderrahmane Kheddar. Generation of whole-body optimal dynamic multi-contact motions. The International Journal of Robotics Research, 32(9-10):1104-1119, 2013.

37. M. Liu, R. Lober, and V. Padois. Whole-body hierarchical motion and force control for humanoid robots. Autonomous Robots, 40(3):493-504, 2016.

38. Mingxing Liu, Alain Micaelli, Paul Evrard, and Adrien Escande. Task-driven posture optimization for virtual characters. In Proceedings of the 11th ACM SIGGRAPH/Eurographics conference on Computer Animation, pages 155-164. Eurographics Association, 2012.

39. Mingxing Liu, Alain Micaelli, Paul Evrard, Adrien Escande, and Claude Andriot. Interactive virtual humans: A two-level prioritized control framework with wrench bounds. Robotics, IEEE Transactions on, 28(6):1309-1322, 2012.

40. Mingxing Liu, Yang Tan, and Vincent Padois. Generalized hierarchical control. Autonomous Robots, 40(1):17-31, 2016.

41. R. Lober, V. Padois, and O. Sigaud. Multiple task optimization using dynamical movement primitives for whole-body reactive control. In Proceedings of the IEEE-RAS International Conference on Humanoid Robots (Humanoids), pages 193-198, Madrid, Spain, November 2014.

42. Ryan Lober, Vincent Padois, and Olivier Sigaud. Variance modulated task prioritization in whole-body control. In Intelligent Robots and Systems (IROS), 2015 IEEE/RSJ International Conference on, pages 3944-3949. IEEE, 2015.

43. N. Mansard, O. Khatib, and A. Kheddar. A unified approach to integrate unilateral constraints in the stack of tasks. IEEE Transactions on Robotics, 25(3):670-685, june 2009.

44. N. Mansard, A. Remazeilles, and F. Chaumette. Continuity of varying-feature-set control laws. IEEE Transactions on Automatic Control, 54(11):2493-2505, 2009.

45. Katja Mombaur. Using optimization to create self-stable human-like running. Robotica, 27(03):321-330, 2009.

46. Igor Mordatch, Emanuel Todorov, and Zoran Popović. Discovery of complex behaviors through contact-invariant optimization. ACM Transactions on Graphics (TOG), 31(4):43, 2012. 
47. Richard M Murray, Zexiang Li, S Shankar Sastry, and S Shankar Sastry. A mathematical introduction to robotic manipulation. CRC PressI Llc, 1994.

48. Christian Ott, Maximo Roa, Gerd Hirzinger, et al. Posture and balance control for biped robots based on contact force optimization. In Humanoid Robots (Humanoids), 2011 11th IEEE-RAS International Conference on, pages 26-33. IEEE, 2011.

49. N. Perrin, D. Lau, and V. Padois. Effective generation of dynamically balanced locomotion with multiple non-coplanar contacts. In International Symposium on Robotics Research (ISRR'15), 2015.

50. Tadej Petrič and Leon Žlajpah. Smooth continuous transition between tasks on a kinematic control level: obstacle avoidance as a control problem. Robotics and Autonomous Systems, 61(9):948-959, 2013.

51. Jerry Pratt, John Carff, Sergey Drakunov, and Ambarish Goswami. Capture point: A step toward humanoid push recovery. In 2006 th IEEE-RAS international conference on humanoid robots, pages 200-207. IEEE, 2006.

52. Jerry E Pratt and Russ Tedrake. Velocity-based stability margins for fast bipedal walking. In Fast Motions in Biomechanics and Robotics, pages 299-324. Springer, 2006.

53. Ludovic Righetti, Jonas Buchli, Michael Mistry, and Stefan Schaal. Inverse dynamics control of floating-base robots with external constraints: A unified view. In Robotics and Automation (ICRA), 2011 IEEE International Conference on, pages 1085-1090. IEEE, 2011.

54. Layale Saab, Oscar E Ramos, François Keith, Nicolas Mansard, Philippe Soueres, and J Fourquet. Dynamic whole-body motion generation under rigid contacts and other unilateral constraints. Robotics, IEEE Transactions on, 29(2):346-362, 2013.

55. Joseph Salini, Vincent Padois, and Philippe Bidaud. Synthesis of complex humanoid whole-body behavior: a focus on sequencing and tasks transitions. In Robotics and Automation (ICRA), 2011 IEEE International Conference on, pages 1283-1290. IEEE, 2011.

56. Benjamin J Stephens and Christopher G Atkeson. Push recovery by stepping for humanoid robots with force controlled joints. In Humanoid Robots (Humanoids), 2010 10th IEEE-RAS International Conference on, pages 52-59. IEEE, 2010.

57. Yuval Tassa, Tom Erez, and Emanuel Todorov. Synthesis and stabilization of complex behaviors through online trajectory optimization. In Intelligent Robots and Systems (IROS), 2012 IEEE/RSJ International Conference on, pages 4906-4913. IEEE, 2012.

58. Miomir Vukobratović and J Stepanenko. On the stability of anthropomorphic systems. Mathematical biosciences, 15(1):1-37, 1972.

59. P.-B. Wieber, R. Tedrake, and S. Kuindersma. Modeling and control of legged robots. In B. Siciliano and O. Khatib, editors, Springer Handbook of Robotics. Springer, 2nd edition, 2015.

60. Pierre-Brice Wieber. On the stability of walking systems. In Proceedings of the international workshop on humanoid and human friendly robotics, 2002.

61. Pierre-Brice Wieber. Trajectory free linear model predictive control for stable walking in the presence of strong perturbations. In Humanoid Robots, 2006 6th IEEE-RAS International Conference on, pages 137-142. IEEE, 2006. 